\title{
Network-wide Consensus Utilizing the Capture Effect in Low-power Wireless Networks
}

\author{
Beshr Al Nahas \\ beshr@chalmers.se \\ Chalmers University of Technology \\ Gothenburg, Sweden
}

\author{
Simon Duquennoy \\ simon.duquennoy@ri.se \\ RISE SICS, Sweden \\ Inria Lille - Nord Europe, France
}

\author{
Olaf Landsiedel \\ olafl@chalmers.se \\ Chalmers University of Technology \\ Gothenburg, Sweden
}

\begin{abstract}
In low-power wireless networking, new applications such as cooperative robots or industrial closed-loop control demand for networkwide consensus at low-latency and high reliability. Distributed consensus protocols is a mature field of research in a wired context, but has received little attention in low-power wireless settings. In this paper, we present $A^{2}$ : Agreement in the Air, a system that brings distributed consensus to low-power multi-hop networks. $A^{2}$ introduces Synchrotron, a synchronous transmissions kernel that builds a robust mesh by exploiting the capture effect, frequency hopping with parallel channels, and link-layer security. $A^{2}$ builds on top of this reliable base layer and enables the two- and threephase commit protocols, as well as network services such as group membership, hopping sequence distribution and re-keying.

We evaluate $A^{2}$ on four public testbeds with different deployment densities and sizes. $A^{2}$ requires only $475 \mathrm{~ms}$ to complete a twophase commit over 180 nodes. The resulting duty cycle is $0.5 \%$ for 1-minute intervals. We show that $A^{2}$ achieves zero losses end-toend over long experiments, representing millions of data points. When adding controlled failures, we show that two-phase commit ensures transaction consistency in $A^{2}$ while three-phase commit provides liveness at the expense of inconsistency under specific failure scenarios.
\end{abstract}

\section{CCS CONCEPTS}

- Networks $\rightarrow$ Network protocol design; Sensor networks;

\section{KEYWORDS}

Consensus, Two-phase commit, Three-phase commit, Group membership, Synchronous transmissions, Capture effect, WSN, IoT

\section{ACM Reference format:}

Beshr Al Nahas, Simon Duquennoy, and Olaf Landsiedel. 2017. Networkwide Consensus Utilizing the Capture Effect in Low-power Wireless Networks. In Proceedings of SenSys '17, Delft, Netherlands, November 6-8, 2017, 14 pages.

DOI: $10.1145 / 3131672.3131685$

\section{INTRODUCTION}

Context and Challenge Many applications in low-power wireless networks build their operation on consensus: For example,

SenSys '17, Delft, Netherlands

(C) 2017 Copyright held by the owner/author(s). Publication rights licensed to ACM This is the author's version of the work. It is posted here for your personal use. Not for redistribution. The definitive Version of Record was published in Proceedings of SenSys '17, November 6-8, 2017, https://doi.org/10.1145/3131672.3131685. networked cooperative robots and UAVs agree on maneuvers to execute [3]; wireless closed-loop control applications such as adaptive tunnel lighting [9] or industrial plants [46, 47] agree on set-points for actuators. Within the network stack, protocols need to agree on which cryptographic keys to use [13], which channel hopping and transmission schedules to follow [30], or which nodes to elect as cluster heads [61].

These application scenarios exhibit key differences when compared to traditional data collection or dissemination in wireless sensor networks: They demand primitives for network-wide consensus at low-latency and highly reliable data delivery with robustness to interference and channel dynamics [2]. For example, after rolling out new cryptographic keys or channel hopping schemes in a network, the new configuration can only be applied once a network-wide agreement has been reached that all nodes are aware of the new data. Otherwise, nodes might be excluded and would need to re-join the network.

Similarly, mission-critical applications such as cooperative robots need to achieve a consensus on which maneuvers to execute. For this, they do not only need to exchange position and velocity information reliably between all nodes at low-latency but also need to know whether the information has been reliably received by all nodes before taking a coordinated action. Specifically, a node $x$ cannot execute an action until it knows that all other nodes agree and in turn, they cannot execute until they know that $x$ agrees with them. This requires multi-phase agreement protocols such as two-phase commit [26]. Protocols for distributed consensus are mature solutions in a wired context, such as for data centers or databases, but have received little attention in low-power wireless settings. We argue that the low latency of new approaches to synchronous transmissions, such as Glossy [23] and Chaos [38], are key enablers for distributed consensus protocols in low-power wireless networks.

Approach In this paper, we tackle the challenge of achieving network-wide consensus in low-power wireless networks. We introduce $A^{2}$ : Agreement in the Air, which builds on a new synchronous transmission kernel, Synchrotron. Synchrotron extends the concepts introduced by Chaos with high-precision synchronization through VHT [53], with time-slotted operation, with a network-wide scheduler, with frequency hopping and multiple parallel channels, and with security features (in part inspired by LWB [21] and TSCH [17, 30]). On top of this robust base layer, we introduce a primitive for network-wide voting, a key component of consensus protocols in $A^{2}$. We then tackle the consensus challenge and show how to enable two- and three-phase commit protocols (2PC and 3PC) $[26,55]$ in low-power wireless settings. In addition, we address the consistent group membership problem and build 
reliable primitives for nodes to join and leave the network. Applications can use $A^{2}$ to reliably agree on, for example, cryptographic keys, channel-hopping sequences or set-points for actuators, even in the presence of node or link failures.

Contributions This paper makes the following contributions:

- We introduce network-wide voting, based on synchronous transmissions;

- We build network-wide consensus protocols for low-power wireless: two- and three-phase commit;

- We devise a consistent group membership protocol based on network-wide transactions;

- We present Synchrotron, $A^{2}$ 's underlying kernel for synchronous transmissions, which provides distributed schedules, the ability to utilize multiple frequencies in parallel, and authentication and encryption to ensure robust and fast agreement;

- We implement and evaluate Synchrotron and $A^{2}$ on four testbeds ranging from 29 to 213 nodes. In our experiments, $A^{2}$ completes a $2 \mathrm{PC}$ round over 180 nodes within $475 \mathrm{~ms}$ at low power. Synchrotron achieves zero losses end-to-end, over millions of data points. Moreover, we evaluate $2 \mathrm{PC}$ and 3PC's liveness and consistency under emulated failures.

Outline We provide the required background on consensus and the Chaos primitive in $\S 2$. In $\S 3$, we outline our primitive for networkwide voting and introduce two- and three-phase commit in $A^{2}$. In $\S 4$, we continue to design a group membership service for $A^{2}$ that lets nodes dynamically join and leave. In $§ 5$, we introduce Synchrotron, our underlying system architecture for synchronous transmissions. In $\S 6$, we evaluate $A^{2}$ and compare it to the state of the art. We discuss related work in $\S 7$ and conclude in $\S 8$.

\section{BACKGROUND}

This section introduces necessary background on the two- and three-phase commit protocols for consensus and on the Chaos communication primitive.

\subsection{Consensus}

Consensus is the problem of reaching agreement among several processes about a proposal, i.e., accept or decline it after a finite time of execution. Achieving consensus becomes challenging when faults may occur, that is, when communication is lossy and processes may crash. Two widely used, yet simple consensus protocols are two-phase commit (2PC) [26] and three-phase commit (3PC) [55] We introduce both next and discuss their respective properties and limitations.

Two-phase commit (2PC) The protocol assumes the existence of one static coordinator and a set of participants, or cohort. As the name suggests, 2PC works in two phases: (a) Proposal Voting: the coordinator broadcasts a proposal to the cohort, each member replies with its vote, yes or no; (b) Decide: the coordinator decides to commit if the vote is yes unanimously; otherwise it decides to abort. It then broadcasts the decision to the cohort that will commit or abort upon receiving the message.

$2 \mathrm{PC}$ is simple and comes at a low communication complexity, but has the major limitation of being a blocking protocol. Whenever a node fails, other nodes will be waiting for its next message or acknowledgment indefinitely, i.e., the protocol may not terminate. Recovery schemes can be considered but fall short when it comes to handling two or more failing nodes. In particular, if the coordinator and a participant both fail during the second phase, other nodes might still be in uncertain state, i.e., have voted yes but not heard the decision from the coordinator. If all remaining nodes are uncertain, they are unable to make a safe decision as they do not know whether the failed nodes had committed or aborted.

Three-phase commit (3PC) Three-phase commit mitigates the above limitations by decoupling decision from commit. This is done with an additional pre-commit phase between the two phases of $2 \mathrm{PC}$. The three phases are as follows: (a) Proposal Voting: same as in 2PC; (b) Pre-Commit (or abort): the coordinator and participants decide as in $2 \mathrm{PC}$, but no commit is applied (abort is applied immediately); (c) Do Commit: participants finally commit. The additional phase guarantees that if any node is uncertain, then no node has proceeded to commit.

The protocol is non-blocking in the case of a single participant node failing: remaining nodes time out and recover independently (commit or abort). 3PC can also handle the failure of the coordinator and multiple nodes, by using a recovery scheme. Nodes will then enter the termination protocol, communicate and unanimously agree to commit, abort, or take over the coordination role and resume operation. In the more challenging case of a network partition, 3PC is, however, unable to maintain consistency.

\subsection{The Chaos Primitive}

Chaos is a primitive for network-wide, all-to-all dissemination, collection, and aggregation. Chaos operates in rounds where nodes send packets synchronously and receive data thanks to the capture effect [39]. In contrast to Glossy and LWB, nodes in Chaos synchronously send different data. At each reception, nodes apply a user-defined in-network aggregation function before sending again in the next slot, until completion of the round.

Synchronous transmissions and capture effect Chaos roots in approaches to synchronous transmissions, such as Glossy, where multiple nodes synchronously transmit the data they want to share. Nodes overhearing the concurrent transmissions receive one of them with high probability, due to the capture effect. For example, to achieve capture with IEEE 802.15.4 radios, nodes need to start transmitting within the duration of the preamble of $160 \mu$ s [38]. Typically, in 802.15.4, the radio receives the stronger one of the synchronous transmissions if its signal is $3 \mathrm{dBm}$ stronger, the socalled co-channel rejection. The exact capture threshold depends on the radio hardware and modulation schemes used.

In-network aggregation In Chaos, each packet contains so-called progress flags, where one bit is assigned to each node in the network. The coordinator node starts a Chaos round by sending a packet with only its own flag set. Upon successful reception, a node sets its flag and merges the received packet with its own. It transmits in the next time slot when it received new information, i.e., new flags, or when it sees that a neighboring node is transmitting messages with fewer flags set, i.e., a neighbor knows less than the node itself. The process continues until all nodes have set their flag. Nodes then enter the so-called final flood phase in which each node transmits 


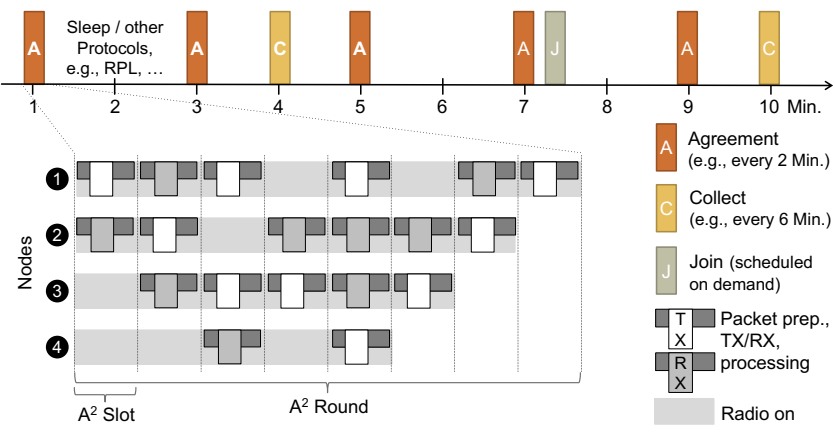

Figure 1: $A^{2}$ Overview: $A^{2}$ schedules rounds for network-wide consensus, dissemination, join, etc. as requested by the application(s). Each round has multiple slots in which nodes transmit, receive or sleep, driven by the transmission policy of the application. Between two rounds, nodes either sleep or are free to run other protocols, such as, for example, RPL.

the result multiple times to disseminate it before completing the round, i.e., turning off. In addition, a timeout mechanism ensures that the network-wide flood is kept alive by re-transmitting when the activity dies during a round.

The rules for merging are application specific. Chaos provides merging rules for network-wide dissemination and data collection. Moreover, it introduces user-defined aggregation functions (the function must be order-insensitive and idempotent). With the Max operation, for example, Chaos identifies the maximum value: Next to the flags, the only payload is the maximum value collected so far. Upon reception, nodes compute the maximum between their local value and the payload, write it to the packet payload, merge the flags, and set their flag before transmitting in the next time-slot.

Chaos is no Consensus Protocol It is important to note that the progress flags set by the nodes throughout a round are not acknowledgment flags. They do not state the recipient of a particular packet; rather, they identify the nodes that contributed their knowledge to the current round. Hence, Chaos does not guarantee reliable reception as nodes may leave a round once they completed their final flood and they have no information whether other nodes also reached their final phase.

$A^{2}$ builds on Chaos: It contributes a voting primitive on top of which it builds consensus primitives and network membership services. $A^{2}$ also contributes at the lower layers, with Synchrotron, exploiting multiple channels, synchronizing more efficiently and ensuring security. $A^{2}$, however, inherits the scaling limitations from Chaos, as it requires a flag bit for each node in the network. Nonetheless, both $A^{2}$ and Chaos easily support a network of a few hundred nodes.

\section{$3 A^{2}$ : AGREEMENT IN THE AIR}

$A^{2}$ proposes a set of network-wide communication primitives:

- Disseminate, Collect and Aggregate are the basic primitives, inherited from Chaos. Respectively, all nodes receive one value from the coordinator, all nodes receive one value from each node, all nodes receive one network-wide aggregate.

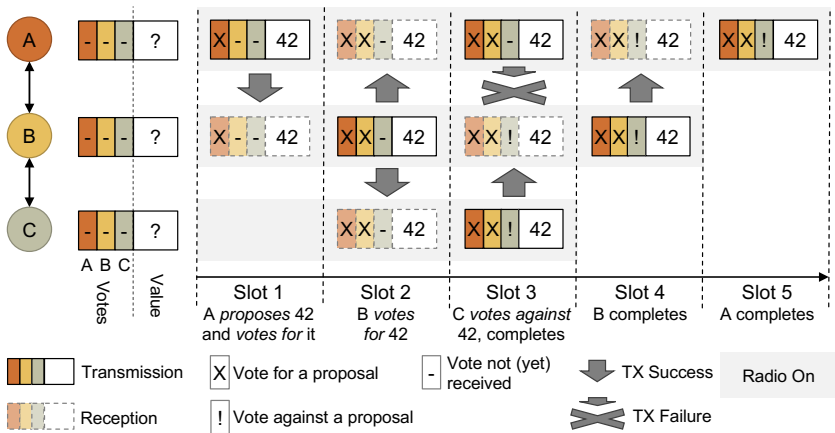

Figure 2: Network-Wide Voting: A initiates a voting round in slot 1 and proposes the value 42. B votes for the proposed value in slot 2. $C$ votes against the value in slot 3 and completes, $B$ completes and relays the packet in slot $4, A$ completes and relays the packet in slot 5. For simplicity, we limit the final flood to one transmission.

- Vote is introduced by $A^{2}$. Nodes vote for or against a coordinator's proposal.

- $2 P C$ is introduced by $A^{2}$. Enables network-wide agreement, with the drawback of being blocking.

- $3 P C$ is introduced by $A^{2}$. Enables network-wide agreement, non-blocking but can result in inconsistencies.

- Multi-phase protocols are introduced by $A^{2}$. Add reliability to Collect and Aggregate.

- Join and Leave are introduced by $A^{2}$. Provide consistent group membership.

We begin by introducing our network-wide voting primitive. Next, we combine it with dissemination and collection primitives as introduced by Chaos to devise protocols for network-wide agreement: two- and three-phase commit. In $\S 4$, we introduce a consistent group membership protocol which dynamically adds and removes participant nodes. $A^{2}$ schedules these primitives according to application requirements, allowing it to multiplex different applications, as illustrated in Figure 1.

\subsection{A Network-Wide Voting Primitive}

We first address the challenge of network-wide voting - a crucial component of $A^{2}$ 's consensus primitives. The voting primitive collects votes from all participants for or against a proposed value. In practice, such a value represents, for example, the ID of an encryption key or a channel hopping sequence. All nodes that can commit to the proposal, because they, for example, have previously received the proposed key or hopping scheme, will vote for it. Any node that cannot commit votes against it. At the end of a successful voting round, the coordinating node is aware of which nodes voted for and against the proposal. For our discussion, we assume the existence of a group membership service within $A^{2}$, i.e., that each node is aware of who else is participating and have been assigned a flag for voting. We introduce such a group membership service in $§ 4$.

Building on the concepts of Chaos, nodes share two pieces of information in each transmission: (a) The proposed value the nodes are voting on and (b) a list of votes representing for each node 
whether it already voted and whether the vote was for or against the proposal. The coordinator begins a round by proposing a value to vote on and by voting for its own proposal, see Figure 2. Upon the first reception during a round, a node votes for or against the proposal and synchronously transmits its vote in the next timeslot. After voting, upon reception, a node merges the received information with any previously received votes of this round. It then transmits in the next time slot if it either (a) learned new information, to spread the new knowledge, or (b) received a packet with fewer votes than it is aware of, i.e., from a neighboring node having less information, aiming to help this neighbor to catch up.

A node reaches completion when all votes are set in its local state. It then proceeds to the final flood stage, i.e., it transmits the final result repeatedly so as to aggressively distribute it in the network, as in Chaos. When a node does not receive for a few slots, a timeout triggers re-transmissions, for resilience to packet losses.

Our voting primitive, just as the original Chaos primitives, is best-effort in nature. As discussed in $§ 2.2$, when a round completes, the only guarantee is that all nodes contributed to the vote/payload. There is no guarantee that all nodes received the final vote/payload, as a node might, for example, fail right after voting but before receiving any other node's vote. To achieve network-wide agreement, we adapt the two- and three-phase commit protocols in $\S 3.2$ and $\S 3.3$.

\subsection{Network-wide Agreement: 2PC}

The two-phase commit protocol (2PC) is the simplest of the two consensus protocols we consider (c.f., §2.1). In $A^{2}$, we achieve $2 \mathrm{PC}$ with two phases of synchronous transmissions back-to-back within a single round. First, in the voting phase, the coordinator proposes a value and collects the cohort's votes. Second, in the commit or abort phase, it disseminates the outcome of the vote, i.e., commit if all agree, or abort otherwise. This is illustrated in Figure 3.

Proposal Voting Phase The nodes vote for or against the proposal. The $2 \mathrm{PC}$ voting phase utilizes the voting primitive introduced in §3.1, but introduces a different termination criterion and behavior. Upon completing the first phase, nodes in the cohort, i.e., non coordinator nodes, do not leave the round as normal after final flood; instead, they continue to transmit the result at random slots ensuring that it spreads and reaches the coordinator eventually.

Commit or Abort Phase Once the result of the voting phase reaches the coordinator, it decides to commit if and only if all nodes accepted the proposal; else, i.e., after a timeout or after receiving one or more votes against its proposal, it aborts. The coordinator spreads the decision using the network-wide dissemination primitive: It commits or aborts, resets the votes, and starts the new phase in the next slot. Upon reception, nodes switch to the new phase, set their flags, adopt the final result and continue the dissemination. This way, the two phases of voting and commit/abort are interleaved rather than strictly segregated, for efficiency.

Failure Handling in 2PC 2PC handles failures with timeout actions or blocking, depending on the phase of the protocol they occurred in. When a node fails before voting yes or after voting no, the coordinator takes a timeout action and aborts the transaction. When it recovers later, the failed node in this stage can safely abort the transaction. Similarly, if a node times out after voting no, it can safely abort. However, if a node times out in the uncertain state, i.e., it voted yes but does not hear back from the coordinator, then it shall block and execute a recovery mechanism later to learn about the outcome of this transaction. Note, a node can commit when it gets the order from the coordinator; i.e., it does not need to block until it is sure that all other nodes get it too. It is important to point out that no further commit can complete until all blocked nodes are recovered.

\subsection{Network-wide Agreement: 3PC}

To overcome the blocking nature of $2 \mathrm{PC}$, where even single-node failures may result in non-termination (c.f., §2.1 and §3.2), we next introduce three-phase commit (3PC) for $A^{2}$. In a nutshell, 3PC adds a buffer state, pre-commit, between the voting and the commit phases, ensuring that no node commits while any other node is still uncertain, i.e., it did cast its vote but is still waiting for the coordinator's decision. In $A^{2}$, this results in three phases executed back-to-back in a round: one vote and two dissemination phases. Note that 3PC trades liveness at the expense safety as discussed in $\S 2.1$.

Proposal Voting Phase The first phase is identical to $2 \mathrm{PC}$, we realize it in $A^{2}$ with our voting primitive. At the end of the phase, the coordinator has collected all votes and decided whether to instruct all participants to commit or abort.

Pre-commit or Abort Phase Once the coordinator has received the votes from all nodes or timed out, it switches to the next phase: If all nodes voted for the proposal, it switches to pre-commit, i.e., to enter the prepared state. Otherwise, it switches to the abort state. The coordinator distributes this new state via the dissemination primitive.

Upon receiving pre-commit, nodes know that the cohort agreed to commit. They prepare for the commit, e.g., lock resources, but do not execute anything that cannot be rolled back. Should any cohort node or network failure occur from this point onward, a timeout will result in the node committing.

This phase uses the $A^{2}$ dissemination primitive: As nodes do not have the option to decline entering prepared or entering abort after voting, no further voting is required. Throughout the round, the coordinator gets individual confirmation through the progress flags, and can hence check whether all nodes have received the information and have transitioned to the requested state, i.e., are all in prepared or abort. At the end of this phase, nodes exit the round if they were instructed to abort, else, they prepare for the final phase.

Do Commit Phase The third and final phase is the commit phase, triggering commit at every node. This phase only follows when all nodes decided to vote for the proposal, i.e., we entered pre-commit and not abort after the voting phase. The coordinator switches to this phase once it knows that all nodes have completed the precommit phase, i.e., moved all to the prepared state. In $A^{2}$, we realize it as dissemination round like the previous pre-commit phase. As all nodes must have confirmed (through setting their flag) the precommit before entering this phase, there is a guarantee that no node is uncertain when nodes commit, a property that enables recovering from multi-node failures. 

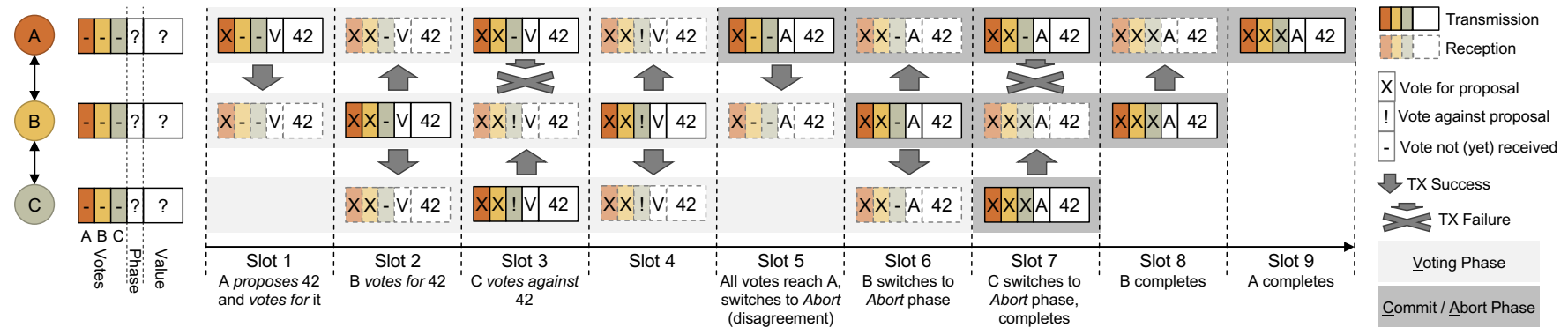

Figure 3: Two-phase commit: Node A proposes value 42 in slot 1. In slot 2, node B agrees by voting for it. In slot 3, node $C$ disagrees by voting against the proposal. This result propagates back to the coordinator, who initiates phase two of 2PC, abort in this example, in slot 5. In the end of the second phase, all nodes have reached the consensus to abort. For simplicity, we limit the final flood to one transmission.

Failure Handling in 3PC We detail here how 3PC handles inconsistencies through timeouts. If a node times out in proposal voting, then it aborts. If a node times out after receiving commit, then it can safely commit. If the coordinator does not receive all flags of precommit after a certain timeout, it concludes that a node has crashed before getting pre-commit, and sends abort to avoid inconsistency. Finally, if a cohort node times out after receiving pre-commit, it concludes that the coordinator crashed in pre-commit, and proceeds to commit. Nodes recovering from a crash are assumed to have a saved state to act on. A recovering node proceeds to commit if it is in pre-commit or commit state, otherwise (voting state) it aborts.

Inconsistencies can happen in the case of network failures, e.g., when a cohort node times out in pre-commit; thus, it proceeds to commit, while the coordinator does not receive its confirmation flag and orders the rest to abort.

\subsection{Multi-Phase Protocols}

Instead of voting for or against a proposal, some applications merely need to reliably collect values or aggregates from the network and take a coordinated action based on this knowledge. Here, we use a modified version of the two-phase design: In the first phase, we use the collection and aggregation primitives to collect the results from all nodes. Once the coordinator has received the final result, it switches to phase two to (a) ensure that no node turns off before the result has reached all and (b) instruct the nodes to take action. Next, we build on this multi-phase design to devise consistent group membership service in $A^{2}$.

\section{$4 A^{2}$ SERVICES}

Building on the primitives introduced in $\S 3$, this section introduces bootstrapping, the join and leave procedures, and services such as channel hopping sequence and key distribution.

\subsection{Bootstrapping: Join Request}

Nodes willing to join the network bootstrap by doing a channel scan. Whenever hearing an $A^{2}$ packet from any node, they synchronize to the network: From the packet header, they learn low-level parameters such as the length of the round, slot size, packet size, and when the next round is scheduled and its application type. Once they are synchronized, they start participating as forwarders only: Upon reception they transmit in the next time slot when they learned new information, i.e., they follow the transmission policy. They do not add own data, as they have not joined the network yet. In addition, they set a single bit flag - the so-called join flag in the packet header of their transmissions. Other nodes receiving this flag during a round also set it in all subsequent transmissions. This flag will eventually propagate in the network and reach the coordinator, which will schedule a join round, detailed next.

\subsection{Join}

The goal of the join operation is to (a) assign a flag index to each node, and (b) inform all existing nodes about the new setup. We realize the join operation as a two-phase protocol. In the first phase we collect the IDs of the new nodes, i.e., nodes wishing to join. The second phase is a dissemination phase in which we disseminate the flag indexes assigned to the joining nodes. The process is illustrated in Figure 4.

Collect Phase The first phase is an extended version of the datacollection primitive, designed to let non-joined nodes contribute to the payload. The coordinator begins the round in the first slot and, upon reception, a node that wishes to join adds its node ID to the list and transmits in the next slot. Already joined nodes set their flag to indicate that they participated, and merge their local state with the received packet. For deterministic merging, we simply sort the IDs added by the nodes wishing to join and eliminate duplicates. Once the list grows too large; i.e., all fields are occupied, the merge operation employs a tiebreaker: We let the highest IDs join by keeping them on the list. Other nodes have to wait for the next round. We are limited by the packet size of 802.15 .4 of 127 bytes, and typically join up to 10 or 20 nodes per round. However, note that the recent $802.15 .4 \mathrm{~g}$ standard supports packets up to $2 \mathrm{kB}$ in certain contexts [30].

Disseminate Phase Since we do not know how many nodes are aiming to join within a particular round, the coordinator cannot in contrast to the previously discussed primitives - use the progress flags to determine when to switch to the dissemination phase, as flags are only assigned to the already joined nodes. Instead, the coordinator switches to the next phase if (a) the node IDs list is full or (b) it does not receive any further updates for a number of slots and the message has propagated through the whole (known) network and back; i.e., all progress flags are set, indicating that all joined nodes have seen it. 

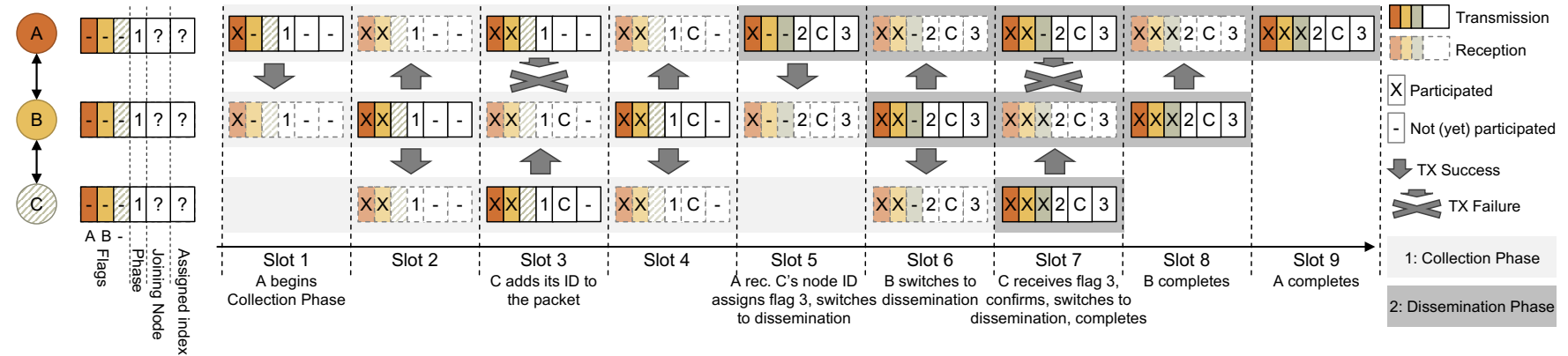

Figure 4: Membership Service: Join. In this example, nodes $A$ and $B$ have already joined and node $C$ wishes to join. Thus, it has not been assigned a flag and our goal is to do so by the end of the round. For simplicity, we only allow one node to join per round here. Node $A$ initiates a join round in slot 1, marks its own flag and sets the phase to one. In slot 2, node B, which already is joined, sets its flag and re-broadcasts. In slot 3 , node $C$ indicates that it would like to join, by writing its node ID C to the payload. It does not set any flags as only joined nodes are assigned flags. In slot 5, the coordinator commits on the new node and assigns a flag ID (the third flag in this example) to it. We mark this in this example, by switching from a patterned filling of the third flag to a solid one. All flags are reset and phase two begins. Upon reception, node $C$ obtains its flag ID and sets its new flag. At the end of the round, all nodes are aware of the new node and the new node $C$ has been assigned the third flag.

When committing on the new nodes, the coordinator assigns a flag index to each new node, registers it in the node list, and starts the dissemination phase by transmitting the list of assigned flags. The new nodes receive their flag indexes and all nodes (both the already joined and the newly joined one) set their flags indicating that have are aware of the new number of nodes. In case a new node misses this dissemination, it will request another join round and it will be assigned the same flag index again to ensure consistency.

A design alternative here is to replace the dissemination with a $2 \mathrm{PC} / 3 \mathrm{PC}$, bringing the join procedure to a total of three/four phases. This ensures all nodes reach a consensus on the flags update. For simplicity, we choose the simpler two-phase design by default, as we are able to resolve any inconsistency at the next round thanks to the sequence numbers introduced in $\S 4.4$.

\subsection{Leave}

If a node does not participate for a number of rounds, the coordinator distributes new flag assignments that remove the node from the list of participants. To remove a node, the coordinator schedules a leave round. Leave is by default a simple dissemination round that spreads the new configuration. As for the join protocol, leave can optionally employ $2 \mathrm{PC} / 3 \mathrm{PC}$, but this is not mandatory as inconsistencies can be fixed at a later point thanks to the sequence numbers, detailed next.

\subsection{Ensuring Consistency: Sequence Numbers}

Once an absent node reconnects to the network, i.e., after it recovers, we have to ensure that it does not continue to participate. To handle consistency despite failure and restart, we assign each configuration a sequence number, increased whenever nodes are added or removed. The coordinator includes it in every packet header to ensure only up-to-date nodes participate. In the case of sequence number mismatch, a node simply requests to join again.

\subsection{Hopping Sequence and Key Distribution}

We use the consensus primitives also for network configuration and maintenance, in particular, to distribute the channel hopping sequence and security keys. This can be done with either $2 \mathrm{PC}$ or 3PC. In the case of success, all nodes will adopt the new hopping sequence or key at the round following the consensus.

\section{SYNCHROTRON}

We introduce Synchrotron, the synchronous transmission kernel we design as a robust lower layer for $A^{2}$. Synchrotron handles packets transmissions and receptions, network synchronization, frequency agility and security aspects, as illustrated in Figure $5 c$ and discussed next. Synchrotron is, in part, inspired by LWB and $\mathrm{TSCH}$ and departs from the design of Chaos; for example, we are not limited to in-network processing operations with a constant number of CPU cycles.

\subsection{Time-Slotted Design and Synchronization}

Synchrotron operates as a time-slotted protocol. The minimum time unit is a slot, which fits one packet transmission/reception and processing, including packet handling by the application, authentication, en- and decryption, etc. Slots are grouped in rounds, where a designated function, such as collect or disseminate is run network-wide, see Figure 1 and Figure $5 b$. Within each slot, a node transmits, receives or sleeps according to the transmission policy of the application.

In-Slot Power Saving In order to accommodate for in-network packet processing, Synchrotron uses slots that last longer than one packet air-time, typically a few milliseconds. Synchrotron disables the radio within the slot after it completes its transmission or reception, see Figure 5a. Moreover, nodes turn their radio off early in case they fail to detect a valid packet at the beginning of the slot. As a result, Synchrotron can efficiently decrease the radio duty-cycle when compared to Chaos.

Synchronization with VHT A key challenge is to keep the nodes synchronized for a complete $A^{2}$ round within the bounds required 


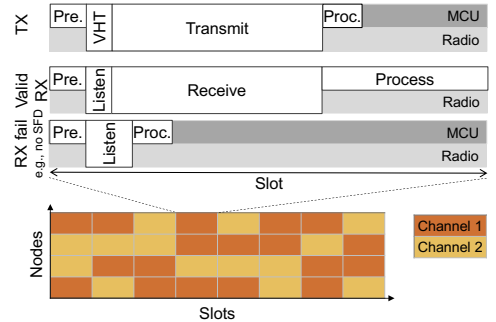

(a) Slotted design of $A^{2}$. Each slot, a node either transmits, aims to receive (or sleeps). The radio is off during processing to reduce the duty-cycle.

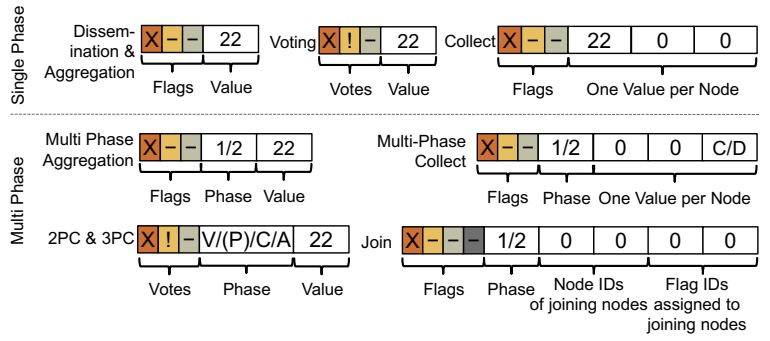

(b) State and packets per application. Each application utilizes a specific set of flags, votes, and fields.

Figure 5: System architecture and details of $A^{2}$.

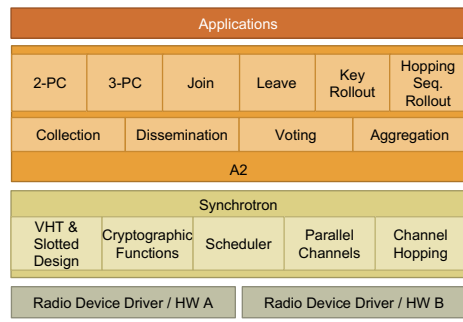

(c) Layered system architecture of $A^{2}$. The scheduler allows $A^{2}$ to operate multiple applications and communication primitives. for successful packet capture (e.g., $160 \mu \mathrm{s})$. A round can last up to several hundred milliseconds and can have phases when the network activity cools down. For example, during a join request (c.f., §4.2), the coordinator commits when it does not receive new information on nodes interested in joining for a number of slots. In such scenarios, we see Chaos losing synchronization, as it depends on the constant activity of transmit/receive.

In Synchrotron, we achieve this synchronization by utilizing VHT: a virtual high-definition timer [53]. VHT uses a combination of the stable, low-power low-frequency clock and the (either unstable or energy-hungry) high-frequency clock of a sensor node. This allows Synchrotron to maintain tight synchronization over long periods of network inactivity. In the context of Glossy, VHT is also employed in recent other work [36, 41, 45].

\subsection{Frequency Agility}

Both Chaos and Glossy see their performance degrade in presence of interference [20,21, 28, 29, 41, 45]. In Synchrotron, we address this by introducing frequency agility and parallel channels.

Channel Hopping for Robustness Nodes in Synchrotron transmit in each time slot on a different frequency following a networkwide schedule, similar to WirelessHART, TSCH or Bluetooth. This prohibits interference on individual channels from disturbing the operation of Synchrotron [59] and allows $A^{2}$ to co-exist with other wireless technologies such as 802.11 or TSCH. Moreover, note that synchronous transmissions in combination with channel hopping have shown their robustness during the EWSN dependability competitions, where, for example, all three top ranking teams in 2017 combine these two design elements [20, 41, 45].

Parallel Channels The probability of capture reduces as the number of concurrent transmitters increases [38]. Chaos proposes reducing the transmission power while in Synchrotron, we use multiple channels in parallel instead. Each node randomly chooses one channel per time-slot to either receive or transmit. As a result, Synchrotron practically reduces network density and thereby increases the probability of capture. Moreover, it does so without increasing the network diameter, as for example, decreasing the transmission power does. As a side effect, this also increases frequency diversity and robustness further. In $\S 6.4$ we show how the number of channels affects performance.

\begin{tabular}{lccccccc}
\hline Testbed & \multicolumn{7}{c}{ Nodes Coordinator Density Diam. Channels Tx Power Flags } \\
& {$[\#]$} & {$[$ [D] } & {$[\#]$} & {$[$ hops] } & {$[\#]$} & {$[$ dBm] } & [bytes] \\
\hline Euratech & 213 & 3 & 106 & 2 & 15 & 0 & 27 \\
Rennes & 180 & 3 & 90 & 2 & 15 & 0 & 23 \\
Indriya & 97 & 35 & 32 & 3 & 2 & 0 & 13 \\
Flocklab & 29 & 3 & 7 & 4 & 2 & 0 & 4 \\
\hline
\end{tabular}

Table 1: The testbeds we used for evaluation. They vary in density from very dense to low-density deployment, and they are subject to real life interference from users and network deployments.

\subsection{Scheduler}

Synchrotron offers a scheduler that allows multiple $A^{2}$ applications to coexist: For example, it can schedule a collect round every 10 seconds, a dissemination round once a minute and schedule on-demand services such as join and leave. The scheduler sets priorities or deadlines (EDF) to each task. In the packet header of each round, Synchrotron denotes the application type, slot length, the maximum allowed number of slots and start time of the next round.

\subsection{Security}

Synchrotron provides security and authentication as standardized by 802.15 .4. We utilize the hardware security support to ensure that the cryptographic operations complete quickly. In our experience, these mechanisms are essential for Synchrotron: For example, when stress-testing the join service, we would occasionally see nodes with invalid node IDs join. Upon investigation, it turned out that this was due to CRC collisions, i.e., corrupted packets which accidentally had valid CRCs. Enabling message authentication in Synchrotron ensures that these faults do not propagate (c.f., §6.3).

\section{EVALUATION}

In this section, we evaluate $A^{2}$ on four public testbeds. We begin by discussing the evaluation setup, then we present a single round of each 3PC and join to highlight the inner workings of $A^{2}$. Next, we evaluate the low-level properties of $A^{2}$ and Synchrotron including reliability, frequency agility, and long-term performance. Finally, we evaluate the performance of the primitives in $A^{2}$ and compare it to the state of the art. 


\subsection{Evaluation Setup}

We present our $A^{2}$ implementation and the scenarios, metrics and testbeds used for evaluation.

Implementation ${ }^{1}$ We implement $A^{2}$ in C for the Contiki OS [16] targeting simple wireless nodes equipped with a low-power radio such as TelosB and Wsn430 platforms which feature a 16bit MSP430 CPU @ $4 \mathrm{MHz}, 10 \mathrm{kB}$ of RAM, $48 \mathrm{kB}$ of firmware storage and CC2420 [57] radio compatible with 802.15.4 [30].

Scenarios We evaluate the following applications:

- Disseminate: shares a 4-byte data item to every node;

- Max: network aggregation where the nodes collectively calculate the maximum value of a 4-byte measurement;

- Collect: collects a 2-byte data item from every node;

- $2 P C$ and $3 P C$ : consensus primitives, as introduced in $\S 3.2$ and $\S 3.3$;

- Glossy Mode: lightweight dissemination (4-byte value) without progress flags, similar to Glossy [23] but run over $A^{2}$;

- LWB: we compare the performance of $A^{2}$ to LWB [21] and LWB-FS [52], which implements forwarder selection as done in CXFS [7].

Depending on the application and testbed, the slot size varies between 3.5 and $7 \mathrm{~ms}$. Unless otherwise mentioned, applications repeat at a 1-minute period. In all experiments, we use a pseudorandom channel-hopping sequence that covers all 16 channels allowed in the IEEE 802.15.4 standard. As a result, we run $A^{2}$ with co-existing 802.11 traffic and other sources such as Bluetooth as our testbeds are deployed in university buildings.

Metrics We focus on the following performance metrics:

- End-to-End Loss Rate: to evaluate how reliable a protocol is. We consider a round reliable only if all nodes receive the final value;

- Radio-on time: is the total time the radio is active during a round, as a proxy for the energy consumed during a round;

- Radio Duty Cycle: is the ratio of the time when the radio is on over the total time, a metric for energy efficiency;

- Latency: is the duration of a round until all nodes complete and they are aware of the final result, i.e., until they enter the final phase.

Testbeds We run our evaluation on four publicly available testbeds deployed in university buildings; namely, Flocklab [40] in ETH, Indriya [11] in NUS, and FIT-IoTLAB [1] in Inria at the sites Rennes and Euratech. The properties of these testbeds are summarized in Table 1, along with the number of parallel channels we use, the transmission power and the length of the $A^{2}$ flags.

\section{2 $A^{2}$ in Action}

We illustrate $A^{2}$ by two representative rounds: three-phase commit and join. We show how the different mechanisms in $A^{2}$ interact when running on large testbeds: Euratech and Rennes.

Three-phase Commit Figure 6 shows a snapshot of a representative 3PC round (c.f., §3.3) in Euratech. All nodes start in phase 0. The coordinator (thick red line) starts the voting phase with a Can commit request in slot 1 . The request disseminates and nodes merge

\footnotetext{
${ }^{1}$ The code is available at: https://github.com/iot-chalmers/a2-synchrotron
}

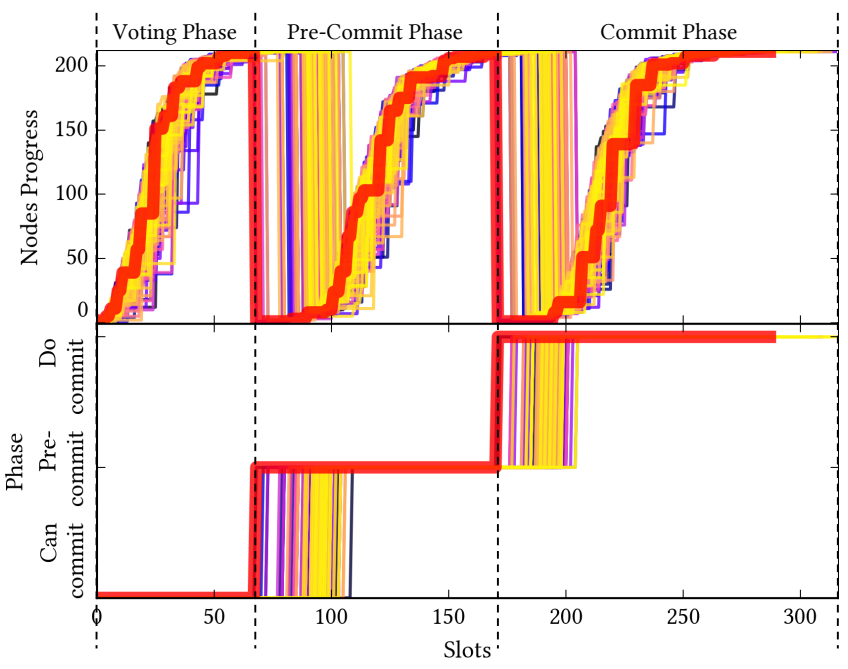

Figure 6: A snapshot of three-phase commit in Euratech. In this example, we show a round of a successful 3PC. The upper figure shows the number of flags each node has set and the lower figure indicates which of the three phases each node is in. The thick red line shows the coordinator, while the other lines belong to the cohort. It takes about 70 slots for the coordinator to receive all votes and to switch to the pre-commit phase. It takes about 30 more slots for the cohort to hear the phase transition. The second and third phases take slightly longer to complete.

their votes, until convergence when all progress/vote flags are set. It takes about 70 slots for the coordinator to receive all votes. In this example, all the votes are positive, the coordinator resets all flags and triggers a transition to the next phase, Pre-commit. It takes about 30 more slots for the cohort to hear the phase transition. We evaluate three-phase commit performance later in $§ 6.6$.

Join We take a close look at the initial joining phase (c.f., §4.2) in the Rennes and Euratech testbeds, with 176 and 212 active nodes respectively. We configure $A^{2}$ to accept 10 new nodes per round. $A^{2}$ shows a stable performance by joining exactly 10 nodes per round. On Rennes, it - in all our experiments - took a stable 18 rounds to join the 176 nodes, while on Euratech it took 22 rounds. Join executes two additional rounds at the end to make sure no nodes were left behind, so in total it took 20 and 24 rounds, respectively, to join all nodes in the networks. When scheduled back to back, this corresponds to a time of 15 and 23 seconds for 176 and 212 nodes, respectively.

Figure 7 shows a snapshot of a representative round in Euratach. At this point, we have successfully joined 100 nodes, and the coordinator starts a new join round. After only 20 time-slots, all 10 join slots are filled by new nodes. Once this information reaches the coordinator, it triggers the next phase and disseminates the number of new nodes and their indices. It takes 30-40 slots for all nodes to transit, and about 80 to complete. After 120 slots in total, the coordinator sees all the flags set, and knows all 10 nodes have joined successfully. It will then switch to a new join round (not shown). 


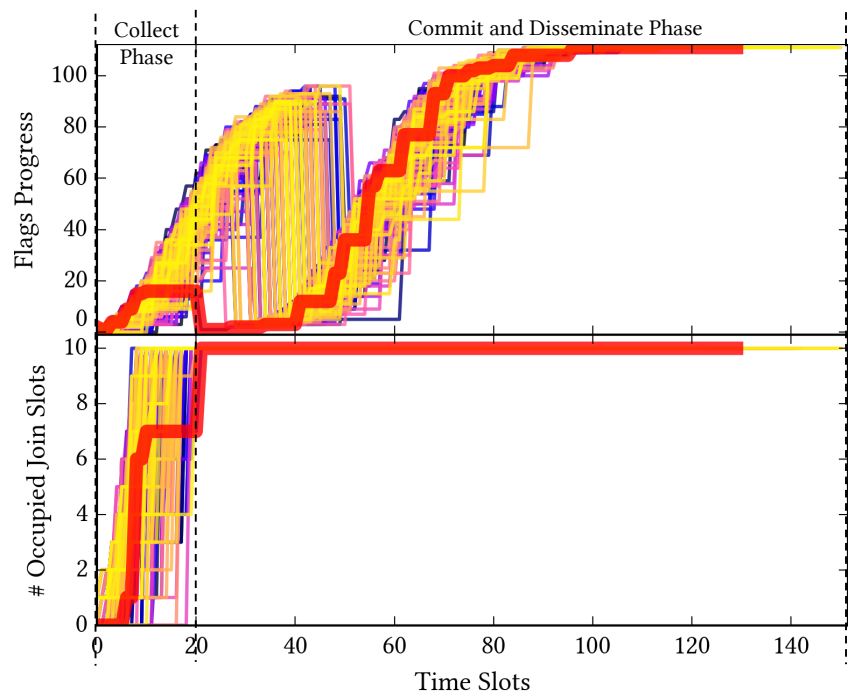

Figure 7: A snapshot of Join in Euratech. In this particular snapshot, we have joined 100 nodes in previous rounds and within this round successfully join 10 further ones. The upper figure shows the number of flags each node has set and the lower figure shows the number of occupied join slots. The thick red line shows the coordinator activity.

\begin{tabular}{lccccc}
\hline Testbed & $\begin{array}{c}\text { Nodes } \\
{[\#]}\end{array}$ & $\begin{array}{c}\text { Rounds } \\
{[\#]}\end{array}$ & $\begin{array}{c}\text { Slot Size } \\
{[\mathrm{ms}]}\end{array}$ & $\begin{array}{c}\text { Slots } \\
{[\#]}\end{array}$ & $\begin{array}{c}\text { Total Time } \\
{[\mathrm{s}]}\end{array}$ \\
\hline Rennes & 176 & 20 & 7 & 107 & 15 \\
Euratech & 212 & 24 & 7 & 137 & 23 \\
\hline
\end{tabular}

Table 2: Summarizing Join performance. Per round, we join up to 10 nodes, limited by the packet size of 802.15.4 and the size of the node IDs, two bytes in this setup. In total, we require 20 and 24 rounds, respectively, and 15 and 23 seconds, respectively to join all nodes.

\begin{tabular}{|c|c|c|c|c|c|c|c|}
\hline Type & $\begin{array}{l}R x \\
\text { Tot. }\end{array}$ & $\begin{array}{l}R x \\
O k\end{array}$ & $\begin{array}{c}R x \\
\text { Corrupt }\end{array}$ & $\begin{array}{l}\text { Tx } \\
\text { Tot. }\end{array}$ & $\begin{array}{c}\text { CRC } \\
\text { Collisions }\end{array}$ & $\begin{array}{l}C R C_{\text {col }} . \\
/ R x_{\text {cor }} .\end{array}$ & $\begin{array}{c}C R C_{\text {col }} . \\
/ R x_{\text {tot }} .\end{array}$ \\
\hline Multiplier & \multicolumn{4}{|c|}{ [packets $\times 10^{6}$ ] } & [packets] & \multicolumn{2}{|c|}{$\left[\right.$ ratio $\left.\times 10^{-6}\right]$} \\
\hline $\mathrm{R}$ & 120 & 103 & 17 & 97 & - & 4. & 0.7 \\
\hline Euratech & 135 & 104 & 31 & 101 & 219 & 7.0 & 1.6 \\
\hline
\end{tabular}

Table 3: CRC collisions happen in Rennes and Euratach. Resp. 4.9e-6 and 7.0e-6 of all corrupted packets suffer a CRC collision, i.e., are wrongly classified as correct at the receiver.

\subsection{Packet Error Detection}

The IEEE 802.15.4 standard provides a 16-bit CRC in the packet footer for transmission error detection. However, in the preliminary evaluation of $A^{2}$, we noticed CRC collisions: Corrupt bytes in the packet that accidentally assemble a correct checksum.

Insights The probability to get a 16-bit CRC collision over corrupted packets is $2^{-16} \approx 15 \times 10^{-6}$, in a simple uniformly distributed random scenario. Our runs include hundreds of million packets,

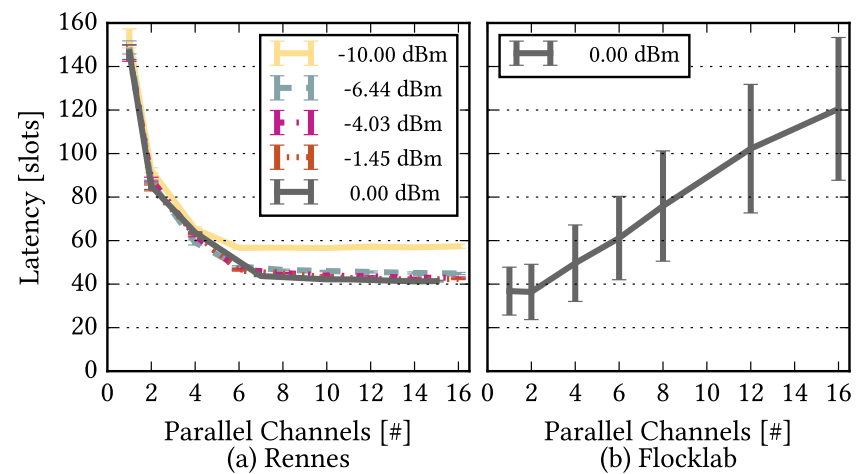

Figure 8: The effect of parallel channels. (a) On a dense testbed, running on 16 channels decreases latency by a factor of roughly 2.53.5, depending on the transmission power. (b) On a sparse testbed, using many channels decreases the performance due to the decreasing probability of finding neighbours on the same channel. Error bars represent the standard deviation.

including several million corrupted packets, in part because $A^{2}$ uses dense transmission schedules to enable capture. This results directly in a number of corrupted packets wrongly classified as correct.

Solution We use the hardware-based AES integrity check provided by IEEE 802.15 .4 radios, not only for its security features but also as an extra guard against packet error. These cryptographic functions are hardware-accelerated and hence fast and energy-efficient. Further, on chips such as the CC2420, the integrity check can even be added/checked on the fly when sending/receiving packets. This results in a constant transmission/reception overhead and hence does not affect the relative synchronization among nodes.

Evaluation We evaluate the number of CRC collisions in our experiments and show how the AES integrity checks (16-bit MIC) improves error detection. The numbers we discuss are with the application Max on Rennes and Euratech, from our long-term experiments presented in more details in $§ 6.5$. We regard a message as corrupted whenever CRC indicates a failure. Conversely, we detect CRC collisions whenever a packet passes the check but fails the AES integrity check. Table 3 shows the packet statistics for experiments in the Rennes and Euratech testbed.

We have over 100 million received packets in each testbed, out of which 17 and 31 million corrupted packets failing the CRC check. Moreover, we notice 84 and 219 broken packets that fail the AES integrity check but pass the CRC check, which we label as CRC collisions. We argue that the additional integrity check is a must since a corrupted packet header could possibly cause arbitrary failures. Further, note that many applications require integrity protection (and sometimes confidentiality), and hence would enable link-layer security regardless.

\subsection{Frequency Agility and Parallel Channels}

We evaluate $A^{2}$ 's parallel channels feature.

Scenario We run the Max application and vary both the transmission power and the number of parallel channels. We cover all 


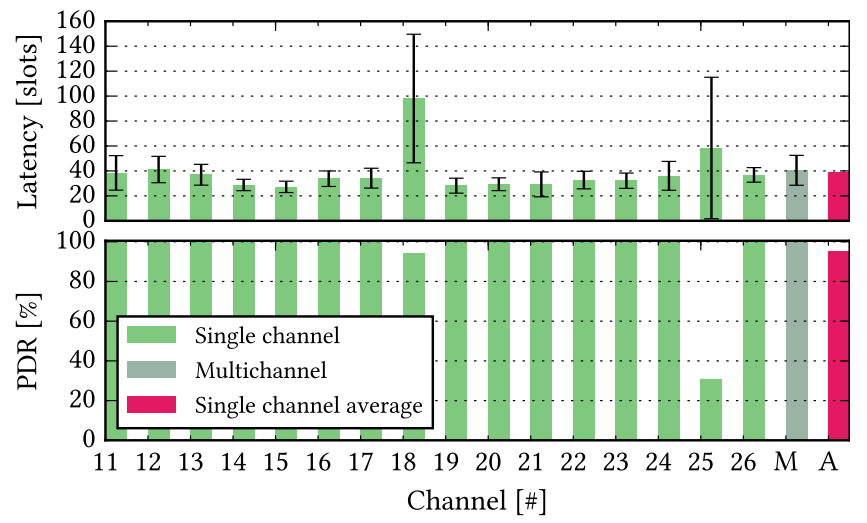

Figure 9: The effect of multichannel on Flocklab. $A^{2}$ channel hopping feature improves reliability while keeping the latency close to the average of the single-channel instances.

testbeds, a number of channels from 1 to 16 , and transmission powers $0,-1.4,-4,-6.4$ and $-10 \mathrm{dBm}$. We measure the resulting latency, i.e., the number of slots to round completion.

Results Figure 8 shows the detailed results in the Rennes and Flocklab testbeds. In a dense testbed (Rennes), the latency drops by half as we enable two channels in parallel, and the trend continues when adding more channels albeit with a less significant drop. The latency converges around 40-60 slots. Overall, we improve performance by a factor of up to 3.5 in Rennes. This is key in enabling the realization of the more complex services such as $2 \mathrm{PC} / 3 \mathrm{PC} /$ join which would take a prohibitively long time to execute otherwise.

A direct side effect of decreased latency is improved energyefficiency, as nodes are equally active regardless of the number of channels. Rounds that are $3.5 \times$ faster also consume roughly $3.5 \times$ less energy.

We also run the same experiments in all other testbeds, to select the optimal settings for all subsequent experiments. Table 1 summarizes the parameters we select. Depending on the testbed, we select 2 to 15 channels and a transmission power of $0 \mathrm{dBm}$. Note that the densest testbeds Rennes and Euratech benefit most from more parallel channels. In sparser testbeds, using too many channels reviewdecreases performance, because nodes have a chance to miss one another (senders and receiver select their channel at random). As a result, we select only two channels in Flocklab and Indriya.

Channel Hopping We do a limited evaluation of the effect of channel hopping on robustness, as this is already a well-understood topic in the literature $[44,54,58]$ and common in today's low-power wireless protocol stacks, such as Bluetooth and TSCH. Figure 9 shows the results in Flocklab testbed. Using multichannel improves reliability and results in a latency close to the average on all channels.

\subsection{Long-Term Performance of $A^{2}$}

In this section, we benchmark the long-term performance of $A^{2}$. We focus on the loss rate to measure the reliability.

\begin{tabular}{lcclccc}
\hline \multirow{2}{*}{ Testbed } & \multicolumn{2}{c}{ Total $\left[10^{6}\right]$} & & \multicolumn{3}{c}{ Average time $[\mathrm{ms}]$} \\
\cline { 2 - 3 } \cline { 5 - 7 } & Points & Losses & & To completion & To off & Radio-on \\
\hline Rennes & 3.87 & 0 & & 151 & 258 & 137 \\
Euratech & 3.72 & 0 & & 203 & 328 & 165 \\
\hline
\end{tabular}

Table 4: Long-term performance of $A^{2}$. No single loss over twice 3.7 million messages delivered end-to-end. Average radio-on time for one round: 137-165 $\mathrm{ms}$.

Scenario We run the application Max on Rennes and Euratech for a total of more than 21000 rounds each. In total, this results in 3.7 million points in each network (number of rounds multiplied by number of nodes). In these experiments, we use slots of $3.75 \mathrm{~ms}$ to accommodate the processing and communication of 56 bytes packets (header, flags, payload and AES MIC). At the time of the experiments, these testbeds had 181 connected nodes each, and in each experiment we got between 178 to 181 nodes working, and 180 on average.

Results Table 4 summarizes the results. First, note that all the rounds in all runs complete, i.e., all the active nodes participate, contribute and receive the final aggregate. We got zero losses in 3.7 million data points over both testbeds within the deadline of the respective rounds. This makes $A^{2}$ suitable for critical applications with high reliability requirements, such as distributed industrial control systems. Note that in other testbeds and applications, we consistently achieve excellent reliability as discussed in $§ 6.6$.

The performance is similar in both testbeds; we focus here on Euratach for the sake of brevity. The average round completion time is $203 \mathrm{~ms}$, i.e., 54 slots, with $3.75 \mathrm{~ms}$ slot length. After completion, nodes initiate the final flood. It takes another 34 slots to reach a stage where all nodes turn their radio off, totaling $328 \mathrm{~ms}$. We give the round a generous deadline of $952.5 \mathrm{~ms}$, i.e., 254 slots. We notice that the nodes finish and turn off well before that. Note that nodes duty cycle their radio within the slots, to accommodate for processing time (c.f., §5.1). As a result, the average radio-on time per round is $165 \mathrm{~ms}$. This is a good indicator for an application to estimate the radio duty cycle required for a Max operation at a given period. A period of 100 seconds would, for example, result in $0.165 \%$ duty cycle.

\subsection{Cost of Consensus}

This section evaluates the performance of network-wide agreement in $A^{2}$.

Scenario We run five applications with increasing degree of consistency; namely, glossy mode i.e., one-to-all with no flags, max and disseminate with all-to-all delivery and flags, two-phase commit and three-phase commit with all-to-all delivery and flags showing the agreement phase and progress, see $\S 6.1$ for details on the evaluated applications. For each experiment, nodes join through the join-primitive of $A^{2}$ then switch to the application; we only focus on application rounds here. We run this evaluation on four testbeds: Flocklab, Indriya, Rennes and Euratech. We run each application for 1200-1700 rounds, except for Indriya (200 rounds), where collecting large amounts of data turned out troublesome due to technical limitations of Indriya. 


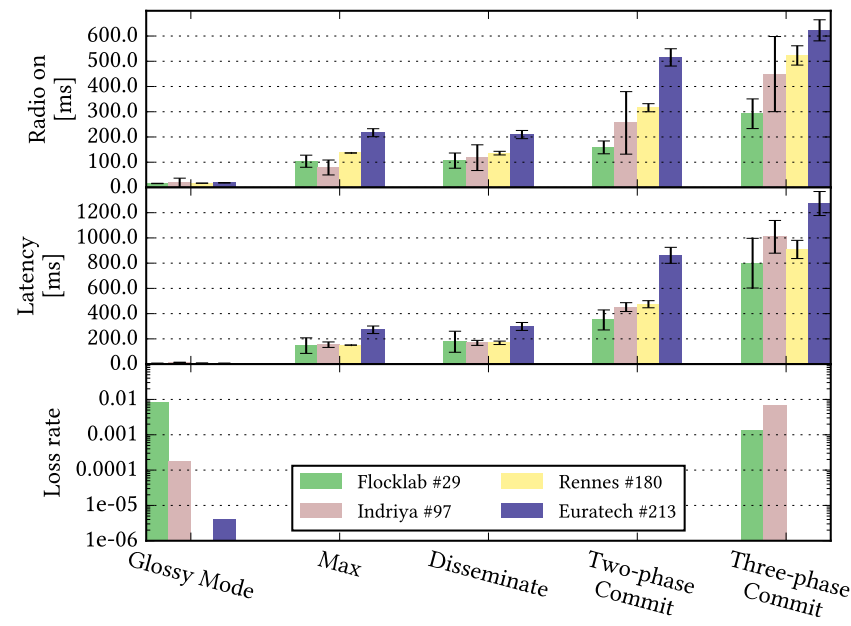

Figure 10: Cost of Consensus in $A^{2}$. The cost increases with the number of nodes, and significantly with the level of consensus. 2PC costs twice as Max and Disseminate, while Glossy Mode is cheap since it does not use flags. Error bars represent the standard deviation.

Results Figure 10 summarizes the loss rate, latency and per-round radio-on time, for each application and testbed. First, we observe a cost increase with the number of nodes in the testbed and with the level of consensus. Note, however, that the cost is not proportional to the testbed size. Rennes is, for example, six times larger than Flocklab but only results in about twice the latency. This due to topology differences, in particular, the higher density in Rennes.

We now take a look at the performance of each application. Glossy Mode has the lowest cost due to its simplicity, but also a relatively high loss rate. Max and Disseminate perform roughly the same since they are very similar. Both achieve zero loss end-to-end. Two-phase commit shows roughly twice the cost of Max (latency and radio-on time). This is due to the two phases and to the packet overhead of vote flags. Three-phase commit continues the trend and has slightly more than three times the cost of Max. This is due to the three phases.We achieve a reliability of $100 \%, 99.9 \%$ and $99 \%$ for 3PC on Rennes, Flocklab and Indriya, respectively. $\$ 6.7$ investigates next the transaction outcome for the consensus primitives.

\subsection{Consistency of Consensus Primitives}

In this section, we evaluate the different consensus primitives in terms of liveness and consistency.

Scenario We compare $2 \mathrm{PC}$ and $3 \mathrm{PC}$, and look at the voting phase in isolation. Note that in $2 \mathrm{PC}$, nodes uncertain about the outcome of the transaction resort to blocking to avoid inconsistencies. 3PC, in contrast, never blocks but can result in some inconsistent outcomes, see $\S 3.2$ and $\S 3.3$.

We run the three primitives on Flocklab for 900 rounds, and inject emulated network failures. At every slot, each node has a given probability (between 0 and $4 \cdot 10^{-5}$ ) to enter a failure state, i.e., be unable to communicate until the end of the round. We do not consider the case where a failed node is stuck, since all the following transactions will be blocked until the Leave operation

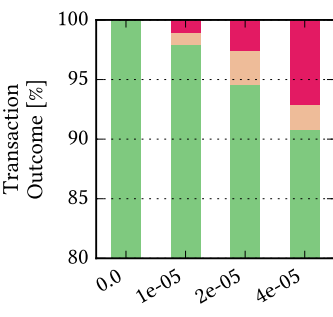

(a) Vote - 1PC

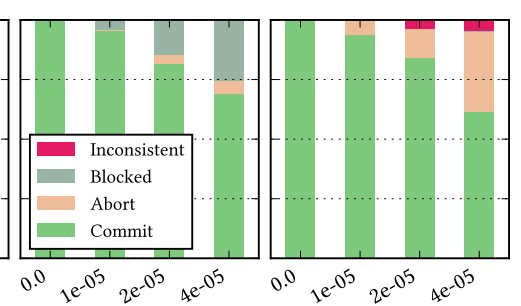

Failure rate
(b) $2 \mathrm{PC}$

(c) $3 \mathrm{PC}$
Figure 11: Consensus outcome of single-phase Vote, 2PC and 3PC in Flocklab under controlled failure. Vote has a low cost but suffers from inconsistencies, $2 P C$ solves inconsistencies with blocking, 3PC trades consistency for liveness and maximizes the ratio of successful (live and consistent) transactions.

kicks in and removes the stuck node. A node that is subject to network failure will decide on the protocol outcome autonomously, based on the timeout/recovery policy of $2 \mathrm{PC}$ and 3PC. This failure model represents either a severe case of network isolation or a crash where the node is able to recover its state from persistent storage. Note that with per-slot probability, protocols that take more slots to finish a transaction (e.g., have more phases) are more vulnerable to failures - which is fair as a slower protocol does have higher chances to suffer a failure during a round in the real world.

Each transaction starts on a clean slate, with all nodes operational. To separate failures and disagreements, we let all nodes vote for the proposal. As a result, abort is merely due to failures and not due to nodes disagreeing to a proposal. We classify the outcome of each transaction as one of the four categories: (a) commit if all nodes commit in the round; (b) abort if all nodes abort as instructed by the protocol, due to a timeout; (c) blocked if one node (and consequently the network) is blocked for this transaction; (d) inconsistent if there are at least one commit and one abort in the same transaction.

Results We summarize the results in Figure 11. First, we notice that all three primitives result in $100 \%$ commit rate in case of no injected failures although we run the experiments on Flocklab and use the 16 channels including those polluted by WiFi and Bluetooth, which underlines the reliability of Synchrotron.

Second, we see that Vote suffers from increasing inconsistencies as the failure rate increases. This is because the voting phase does not offer protection against the case that one node votes yes and fails to hear the rest of the votes; thus aborts due to timeout, while the rest of the nodes are not aware of its abort as they all voted yes, heard its yes vote and committed.

Third, we notice that $2 P C$ delivers on the promise of eliminating the inconsistencies, as it orders the nodes that are in the uncertainty state to block (i.e., those who voted yes and failed to hear the outcome). The price to pay is liveness, as the whole protocol has to block until all nodes are available again for recovery.

Finally, $3 P C$ results in few inconsistencies in favor of liveness. Note that it has a significantly higher rate of successful transactions (live and consistent) than Vote or 2PC. We conclude that for applications that cannot afford inconsistencies, then $2 \mathrm{PC}$ is a safe 


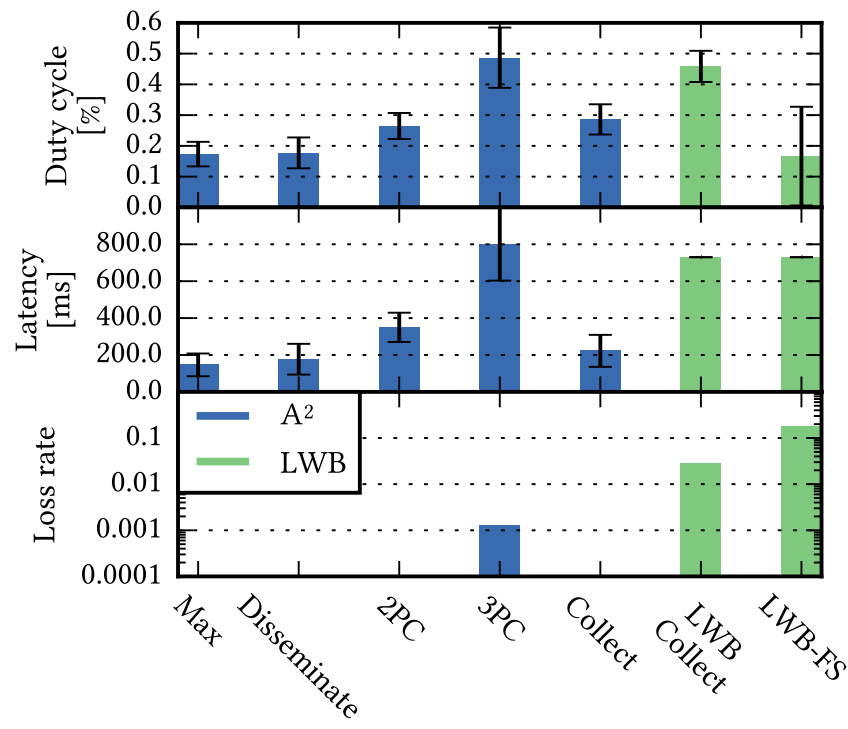

Figure 12: Performance comparison between $A^{2}$ and LWB(-FS) in Flocklab. Period: 1 minute, payload: 2 bytes. $A^{2}$ outperforms the state of the art in terms of latency and reliability. LWB-FS achieves the lowest duty cycles but is also the least reliable solution.

solution. For applications that cannot afford to block but can do with little inconsistencies then $3 \mathrm{PC}$ is a practical option.

\subsection{Comparison to the State of the Art}

We evaluate the performance of the different applications implemented over $A^{2}$ and compare them to the state of the art: the Lowpower Wireless Bus (LWB) and LWB with forwarder selection (FS). For a description of the applications see $§ 6.1$.

Scenario We limit this evaluation to Flocklab, as the other testbeds are not supported in the LWB code base. The comparison between $A^{2}$, LWB and LWB-FS is on Collect, but we also show $A^{2}$ 's other primitives for reference. We scale the duty-cycle calculation to reflect a rate of one packet per minute per node to emulate an IoT data collection scenario, e.g., building monitoring or climate control, and we use a payload of two bytes. As the network consists of 29 nodes, the collect payload in $A^{2}$ is 58 bytes (the packet needs enough space for a value from each node). Note that the implementations of LWB and LWB-FS do not support multi-channel operation and our experiments are limited to channel 26. Thus, these two protocols do not employ frequency agility as $A^{2}$ does.

Results We summarize the results in Figure 12. For $A^{2}$, we notice that Collect has a latency and duty cycle slightly over Disseminate and Max, because Collect has a larger payload.

LWB Collect has a higher duty cycle of $0.45 \%$ vs. $0.29 \%$ in $A^{2}$, a higher latency of 730 vs. $222 \mathrm{~ms}$, and a significant loss-rate of $2.82 \%$. The higher loss-rate can be explained by two factors: (a) LWB uses a single channel, which makes it vulnerable to interference; and (b) LWB nodes do not wait for flags from the sink. Instead, they use a fixed number of transmissions, which has the benefit to yield a fixed latency. We note that the higher latency with the default settings of LWB could be significantly lowered: By taking a $6 \mathrm{~ms}$ data slot for each of the 29 nodes and a $10 \mathrm{~ms}$ contention slot, the latency can be as low as $190 \mathrm{~ms}$ per one LWB round, and thereby be comparable to $A^{2}$ in terms of latency. The loss rate, however, would be unaffected.

With its forwarder selection feature, LWB-FS is able to dynamically turn off the nodes off the path between source and sink. This makes LWB-FS the most energy-efficient solution, but also the least reliable one, as there are fewer nodes participating in the flood. Overall, $A^{2}$ is by far the most reliable, it has the lowest latency but a worse duty cycle than LWB-FS.

We discuss the potential cost of a hypothetical 2PC solution on top of LWB: Such a solution would require at least two Collect rounds, as each phase involves getting a vote/ack from the cohort. This would lead to a latency above $1400 \mathrm{~ms}$, that is, slower than $A^{2}$ (with default settings) by a factor 6 .

\section{RELATED WORK}

In this section, we discuss the broader field of synchronous transmissions and advanced communication primitives in wireless sensor networks (WSNs).

Synchronous Transmissions Glossy [23] pioneered the field of synchronous (or concurrent) transmissions in WSNs. In Glossy, nodes synchronously transmit the same data packet. Tight synchronization ensures non-destructive interference of the transmissions and allows receivers to recover the packet.

Glossy provides network-wide flooding, i.e., from a single sender to all nodes in the network, on millisecond scale. Others, such as LWB [21], Splash [12], Choco [56] base on Glossy to schedule individual network-floods to provide data collection. Virtus builds on Glossy to provide virtual synchrony [4] and Crystal [31] relies on data prediction to reduce the number of Glossy floods. CXFS [7], Sparkle [63] and others [6, 33, 52, 62, 64] limit the number of concurrent transmitters in Glossy or LWB to improve energy efficiency.

In contrast to Chaos and $A^{2}$, nodes in Glossy always need to transmit the same data packet to enable constructive interference. As a result, they cannot exploit spatial diversity as Chaos and $A^{2}$ do. Moreover, their tight timing requirements of constructive interference limit the options of in-network processing of data. Overall, synchronous transmissions enable low-latency network-wide communication. $A^{2}$ builds on these results and provide advanced communication primitives.

Conventional Networking Protocols Conventional asynchronous protocols such as ContikiMAC [15] or even newer TDMA approaches such as TSCH $[17,30]$ and Orchestra [18] when combined with routing protocols such as RPL [60], CTP [25] provide best-effort low-power routing. WSN rate-controlled protocols [14, 32, 35, 49, 51] or TCP for 6LoWPAN [19] enable end-to-end reliability on top of a best-effort routing protocol. These protocols have significant higher latencies than $A^{2}$ and other approaches based on synchronous transmissions [21,38].

Further, supporting group communication on top of a routing protocol is a challenge per se. Solutions such as BMRF [24] or SMRF [48] provide such feature in a RPL multicast context, but 
they exhibit high latencies and loss rates of a few percent or tens of percent. Most importantly, existing group communication protocols are best-effort, i.e., they do not provide end-to-end reliability. The lack of end-to-end acknowledgments combined with the often significantly higher latency make it very challenging to realize advanced communication primitives as provided by $A^{2}$.

Network-wide Agreement and Transactions Achieving agreement in conventional distributed systems is a mature research field, with solutions such as 2PC [26] and 3PC [55], or PBFT [8] for the even more challenging context of Byzantine Fault Tolerance. Wireless sensor networks, however, bring unique challenges, in particular the low-power, multi-hop nature of the network, and the lossiness of the links.

Existing literature covers distributed data processing and aggregation [34] in WSN, but more demanding primitives such as atomic transactions, fault tolerant consensus, and reliable multicast are, today, limited to simulation or modeling [27, 37, 42, 43]. Practical approaches are limited to single-hop networking $[5,10,50]$ or have a latency of several seconds [22]. For example, JAG [5] provides reliable agreement between pairs of neighbors with a focus on interference resilience but does not address network-wide agreement. The $6 \mathrm{P}$ protocol [50] is being designed as part of IETF $6 \mathrm{TiSCH}$ for schedule negotiation; it offers transaction between pairs of neighbors based on 2/3-way handshake. In contrast, $A^{2}$ builds on $2 \mathrm{PC}$ and 3PC to provide network-wide transactions in WSN, over hundreds of nodes, at low-latency and high reliability.

\section{CONCLUSION}

This paper introduces low-latency and reliable consensus in lowpower wireless networks. We argue that new approaches to synchronous transmissions, such as Glossy and Chaos, are key enablers for such protocols. We present $A^{2}$ : a network stack based on the capture effect, that offers advanced communication primitives, such as network-wide consensus with two- and three-phase commit and consistent group membership. $A^{2}$ supports frequency diversity and exploits multiple radio channels simultaneously to increase reliability and reduce latency. Our extensive experimental evaluation shows that $A^{2}$ (a) is highly reliable with no losses over millions of points; (b) achieves low power and low latency; and (c) enables network-wide agreement, with different consistency/liveness tradeoffs.

\section{ACKNOWLEDGMENTS}

We would like to thank the Computer Engineering and Networks Laboratory in ETH, Zurich for providing the Flocklab testbed, the CIR Lab in Singapore for providing the Indriya testbed, and the FIT consortium for providing the IoT-Lab testbeds Rennes and Euratech. Also, we would like to thank Omprakash Gnawali, our shepherd, for his insightful comments. This work was partly supported by the Swedish Research Council (VR) through the project ChaosNet, the Swedish Foundation for Strategic Research (SSF) through the project LoWi, CPER Nord-Pas-de-Calais/FEDER DATA and Sweden's innovation agency (VINNOVA).

\section{REFERENCES}

[1] Cedric Adjih, Emmanuel Baccelli, Eric Fleury, Gaetan Harter, Nathalie Mitton, Thomas Noel, Roger Pissard-Gibollet, Frederic Saint-Marcel, Guillaume Schreiner,
Julien Vandaele, and others. 2015. FIT IoT-LAB: A large scale open experimental IoT testbed. In IEEE 2nd World Forum on Internet of Things (WF-IoT).

[2] Johan Åkerberg, Mikael Gidlund, Tomas Lennvall, Krister Landerns, and Mats Bjökman. 2013. Design Challenges and Objectives in Industrial Wireless Sensor Networks. In Industrial Wireless Sensor Networks: Applications, Protocols, and Standards.

[3] Jude Allred, Ahmad Bilal Hasan, Saroch Panichsakul, William Pisano, Peter Gray, Jyh Huang, Richard Han, Dale Lawrence, and Kamran Mohseni. 2007. SensorFlock: An Airborne Wireless Sensor Network of Micro-air Vehicles. In Proceedings of the Conference on Embedded Networked Sensor Systems (ACM SenSys).

[4] Kenneth P. Birman. 1993. The Process Group Approach to Reliable Distributed Computing. Commun. ACM 36, 12 (1993).

[5] C. A. Boano, M. A. Zuniga, K. Römer, and T. Voigt. 2012. JAG: Reliable and Predictable Wireless Agreement under External Radio Interference. In Proceedings of the IEEE Real-Time Systems Symposium (IEEE RTSS).

[6] M. Brachmann, O. Landsiedel, and S. Santini. 2016. Concurrent Transmissions for Communication Protocols in the Internet of Things. In Proceedings of the Conference on Local Computer Networks (IEEE LCN).

[7] Douglas Carlson, Marcus Chang, Andreas Terzis, Yin Chen, and Omprakash Gnawali. 2013. Forwarder selection in multi-transmitter networks. In Proceedings of the Conference Distributed Computing in Sensor Systems (DCOSS).

[8] Miguel Castro and Barbara Liskov. 1999. Practical Byzantine Fault Tolerance. In Proceedings of the Symposium on Operating Systems Design \& Implementation (USENIX OSDI).

[9] M. Ceriotti, M. Corrà, L. D’Orazio, R. Doriguzzi, D. Facchin, S. T. Gună, G. P. Jesi, R. L. Cigno, L. Mottola, A. L. Murphy, M. Pescalli, G. P. Picco, D. Pregnolato, and C. Torghele. 2011. Is there light at the ends of the tunnel? Wireless sensor networks for adaptive lighting in road tunnels. In Proceedings of the Conference on Information Processing in Sensor Networks (ACM/IEEE IPSN).

[10] M. Demirbas, O. Soysal, and M. Hussain. 2008. TRANSACT: A Transactional Framework for Programming Wireless Sensor/Actor Networks. In Proceedings of the Conference on Information Processing in Sensor Networks (ACM/IEEE IPSN).

[11] Manjunath Doddavenkatappa, Mun Choon Chan, and A.L. Ananda. 2011. Indriya: A Low-Cost, 3D Wireless Sensor Network Testbed. In Proceedings of the Cginonference on Testbeds and Research Infrastructures for the Development of Networks \& Communities (TridentCom).

[12] Manjunath Doddavenkatappa, Mun Choon Chan, and Ben Leong. 2013. Splash: Fast Data Dissemination with Constructive Interference in Wireless Sensor Networks. In Proceedings of the Symposium on Networked Systems Design \& Implementation (USENIX NSDI).

[13] Wenliang Du, Jing Deng, Yunghsiang S. Han, Pramod K. Varshney, Jonathan Katz, and Aram Khalili. 2005. A Pairwise Key Predistribution Scheme for Wireless Sensor Networks. ACM Trans. Inf. Syst. Secur. (2005).

[14] Wan Du, Jansen Christian Liando, Huanle Zhang, and Mo Li. 2015. When Pipelines Meet Fountain: Fast Data Dissemination in Wireless Sensor Networks. In Proceedings of the Conference on Embedded Networked Sensor Systems (ACM SenSys).

[15] Adam Dunkels. 2011. The ContikiMAC Radio Duty Cycling Protocol. Technical Report T2011:13. Swedish Institute of Computer Science.

[16] Adam Dunkels, Bjorn Gronvall, and Thiemo Voigt. 2004. Contiki - A Lightweight and Flexible Operating System for Tiny Networked Sensors. In Proceedings of the Conference on Local Computer Networks (IEEE LCN).

[17] Simon Duquennoy, Atis Elsts, Beshr Al Nahas, and George Oikonomou. 2017. TSCH and 6TiSCH for Contiki: Challenges, Design and Evaluation. In Proceedings of the Conference Distributed Computing in Sensor Systems (DCOSS).

[18] Simon Duquennoy, Beshr Al Nahas, Olaf Landsiedel, and Thomas Watteyne. 2015. Orchestra: Robust Mesh Networks Through Autonomously Scheduled TSCH. In Proceedings of the Conference on Embedded Networked Sensor Systems (ACM SenSys).

[19] Simon Duquennoy, Fredrik Österlind, and Adam Dunkels. 2011. Lossy Links, Low Power, High Throughput. In Proceedings of the Conference on Embedded Networked Sensor Systems (ACM SenSys).

[20] Antonio Escobar, Javier Garcia, Francisco Cruz, Jirka Klaue, Angel Corona, and Divya Tati. 2017. Competition: RedFixHop with Channel Hopping. In Proceedings of the European Conference on Wireless Sensor Networks (EWSN).

[21] F. Ferrari, M. Zimmerling, L. Mottola, and L. Thiele. 2012. Low-Power Wireless Bus. In Proceedings of the Conference on Embedded Networked Sensor Systems (ACM SenSys).

[22] F. Ferrari, M. Zimmerling, L. Mottola, and L. Thiele. 2013. Virtual Synchrony Guarantees for Cyber-physical Systems. In Proceedings of the IEEE International Symposium on Reliable Distributed Systems (IEEE SRDS).

[23] Federico Ferrari, Marco Zimmerling, Lothar Thiele, and Olga Saukh. 2011. Efficient Network Flooding and Time Synchronization with Glossy. In Proceedings of the Conference on Information Processing in Sensor Networks (ACM/IEEE IPSN).

[24] Guillermo Gastón Lorente, Bart Lemmens, Matthias Carlier, An Braeken, and Kris Steenhaut. 2017. BMRF: Bidirectional Multicast RPL Forwarding. Ad Hoc Netw. 54 (1 2017). 
[25] Omprakash Gnawali, Rodrigo Fonseca, Kyle Jamieson, David Moss, and Philip Levis. 2009. Collection Tree Protocol. In Proceedings of the Conference on Embedded Networked Sensor Systems (ACM SenSys).

[26] Jim Gray. 1978. Notes on Data Base Operating Systems. In Operating Systems, An Advanced Course.

[27] G. Gupta and M. Younis. 2003. Fault-tolerant clustering of wireless sensor networks. In Proceddings of the IEEE Conference on Wireless Communications and Networking (WCNC).

[28] Z. He, K. Hewage, and T. Voigt. 2016. Arpeggio: A Penetration Attack on Glossy Networks. In Proceedings of the Conference on Sensor, Mesh and Ad Hoc Communications and Networks (IEEE SECON).

[29] Kasun Hewage, Shahid Raza, and Thiemo Voigt. 2015. An experimental study of attacks on the availability of Glossy. Computers \& Electrical Engineering 41 (2015).

[30] IEEE. 2016. 802.15.4-2015: IEEE Standard for Local and metropolitan area networks-Part 15.4: Low-Rate Wireless Personal Area Networks (LR-WPANs) 1: MAC sublayer. (22 April 2016).

[31] Timofei Istomin, Amy L. Murphy, Gian Pietro Picco, and Usman Raza. 2016. Data Prediction + Synchronous Transmissions = Ultra-low Power Wireless Sensor Networks. In Proceedings of the Conference on Embedded Networked Sensor Systems (ACM SenSys).

[32] Yogesh G. Iyer, Shashidhar Gandham, and S. Venkatesan. 2005. STCP: A Generic Transport Layer Protocol for Wireless Sensor Networks. In Proceedings of the IEEE International Conference on Computer Communications and Networks (IEEE ICCCN).

[33] J. Jeong, J. Park, H. Jeong, J. Jun, C. J. M. Liang, and J. Ko. 2014. Low-power and topology-free data transfer protocol with synchronous packet transmissions. In Proceedings of the Conference on Sensor, Mesh and Ad Hoc Communications and Networks (IEEE SECON).

[34] Shouling Ji, Jing (Selena) He, and Zhipeng Cai. 2014. Data Gathering in Wireless Sensor Networks. In The Art of Wireless Sensor Networks, Habib M. Ammari (Ed.). Springer.

[35] Sukun Kim, Rodrigo Fonseca, Prabal Dutta, Arsalan Tavakoli, David Culler, Philip Levis, Scott Shenker, and Ion Stoica. 2007. Flush: A Reliable Bulk Transport Protocol for Multihop Wireless Networks. In Proceedings of the Conference on Embedded Networked Sensor Systems (ACM SenSys).

[36] Michael König and Roger Wattenhofer. 2016. Maintaining Constructive Interference Using Well-Synchronized Sensor Nodes. In Proceedings of the Conference Distributed Computing in Sensor Systems (DCOSS).

[37] B. Krishnamachari and S. Iyengar. 2004. Distributed Bayesian algorithms for fault-tolerant event region detection in wireless sensor networks. IEEE Trans. Comput. 53, 3 (2004).

[38] Olaf Landsiedel, Federico Ferrari, and Marco Zimmerling. 2013. Chaos: Versatile and Efficient All-to-All Data Sharing and In-Network Processing at Scale. In Proceedings of the Conference on Embedded Networked Sensor Systems (ACM SenSys).

[39] K. Leentvaar and J. Flint. 1976. The Capture Effect in FM Receivers. IEEE Trans. Commun. 24, 5 (1976)

[40] R. Lim, F. Ferrari, M. Zimmerling, C. Walser, P. Sommer, and J. Beutel. 2013 FlockLab: A Testbed for Distributed, Synchronized Tracing and Profiling of Wireless Embedded Systems. In Proceedings of the Conference on Information Processing in Sensor Networks (ACM/IEEE IPSN).

[41] Roman Lim, Reto Da Forno, Felix Sutton, and Lothar Thiele. 2017. Competition Robust Flooding using Back-to-Back Synchronous Transmissions with ChannelHopping. In Proceedings of the European Conference on Wireless Sensor Networks (EWSN).

[42] Hai Liu, Amiya Nayak, and Ivan Stojmenović. 2009. Fault-Tolerant Algorithms/Protocols in Wireless Sensor Networks.

[43] X. Luo, M. Dong, and Y. Huang. 2006. On distributed fault-tolerant detection in wireless sensor networks. IEEE Trans. Comput. 55, 1 (2006).

[44] Beshr Al Nahas, Simon Duquennoy, Venkatraman Iyer, and Thiemo Voigt. 2014 Low-Power Listening Goes Multi-Channel. In Proceedings of the Conference Distributed Computing in Sensor Systems (DCOSS).
[45] Beshr Al Nahas and Olaf Landsiedel. 2017. Competition: Towards Low-Power Wireless Networking that Survives Interference with Minimal Latency. In Proceedings of the European Conference on Wireless Sensor Networks (EWSN).

[46] V. Navda, A. Bohra, S. Ganguly, and D. Rubenstein. 2007. Using Channel Hopping to Increase 802.11 Resilience to Jamming Attacks. In Proceedings of the Conference on Computer Communications (IEEE INFOCOM)

[47] Tony O'donovan, James Brown, Felix Büsching, Alberto Cardoso, José Cecílio, Jose Do Ó, Pedro Furtado, Paulo Gil, Anja Jugel, Wolf-Bastian Pöttner, Utz Roedig, Jorge Sá Silva, Ricardo Silva, Cormac J. Sreenan, Vasos Vassiliou, Thiemo Voigt, Lars Wolf, and Zinon Zinonos. 2013. The GINSENG System for Wireless Monitoring and Control: Design and Deployment Experiences. ACM Trans. Sen. Netw. 10, 1 (2013).

[48] G. Oikonomou and I. Phillips. 2012. Stateless multicast forwarding with RPL in 6LowPAN sensor networks. In IEEE International Conference on Pervasive Computing and Communications, Workshops.

[49] Jeongyeup Paek and Ramesh Govindan. 2010. RCRT: Rate-controlled Reliable Transport Protocol for Wireless Sensor Networks. ACM Trans. Sen. Netw. 7, 3 (2010).

[50] Q. Wang and others. 2017. 6top Protocol (6P). (2017). IETF draft-ietf-6tisch-6topprotocol-04, WiP.

[51] Sumit Rangwala, Ramakrishna Gummadi, Ramesh Govindan, and Konstantinos Psounis. 2006. Interference-aware Fair Rate Control in Wireless Sensor Networks. SIGCOMM Comput. Commun. Rev. 36, 4 (2006).

[52] Chayan Sarkar. 2016. LWB and FS-LWB implementation for Sky nodes using Contiki. arXiv preprint. (2016). https://arxiv.org/pdf/1607.06622.pdf

[53] Thomas Schmid, Prabal Dutta, and Mani B. Srivastava. 2010. High-resolution, Low-power Time Synchronization an Oxymoron No More. In Proceedings of the Conference on Information Processing in Sensor Networks (ACM/IEEE IPSN).

[54] Markus Schuß, Carlo Alberto Boano, Manuel Weber, and Kay Uwe Römer. 2017. A Competition to Push the Dependability of Low-Power Wireless Protocols to the Edge. In Proceedings of the European Conference on Wireless Sensor Networks (EWSN).

[55] D. Skeen and M. Stonebraker. 1983. A Formal Model of Crash Recovery in a Distributed System. IEEE Transactions on Software Engineering SE-9, 3 (1983).

[56] M. Suzuki, Y. Yamashita, and H. Morikawa. 2013. Low-Power, End-to-End Reliable Collection Using Glossy for Wireless Sensor Networks. In IEEE 77th Vehicular Technology Conference (VTC Spring).

[57] Texas Instruments. 2006. Chipcon CC2420: 2.4 GHz IEEE 802.15.4 / ZigBee-ready RF Transceiver. (2006)

[58] T. Watteyne, S. Lanzisera, A. Mehta, and K.S.J. Pister. 2010. Mitigating Multipath Fading through Channel Hopping in Wireless Sensor Networks. In Proceedings of the IEEE International Conference on Communications (IEEE ICC).

[59] Thomas Watteyne, Ankur Mehta, and Kris Pister. 2009. Reliability Through Frequency Diversity: Why Channel Hopping Makes Sense. In Proceedings of the ACM Symposium on Performance Evaluation of Wireless Ad Hoc, Sensor, and Ubiquitous Networks (PE-WASUN).

[60] T. Winter, P. Thubert, A. Brandt, J. Hui, R. Kelsey, P. Levis, K. Pister, R. Struik, J. Vasseur, and R. Alexander. 2012. RPL: IPv6 Routing Protocol for Low-Power and Lossy Networks. RFC 6550 (Proposed Standard). (2012).

[61] Mao Ye, Chengfa Li, Guihai Chen, and J. Wu. 2005. EECS: an energy efficient clustering scheme in wireless sensor networks. In IEEE International Performance, Computing, and Communications Conference (PCCC).

[62] D. Yuan and M. Hollick. 2015. Ripple: High-throughput, reliable and energyefficient network flooding in wireless sensor networks. In Proceedings of the Symposium on a World of Wireless Mobile and Multimedia Networks (IEEE WoWMoM).

[63] Dingwen Yuan, Michael Riecker, and Matthias Hollick. 2014. Making 'Glossy' Networks Sparkle: Exploiting Concurrent Transmissions for Energy Efficient, Reliable, Ultra-Low Latency Communication in Wireless Control Networks. In Proceedings of the European Conference on Wireless Sensor Networks (EWSN).

[64] Jin Zhang, Andreas Reinhardt, Wen Hu, and Salil S. Kanhere. 2015. RFT: Identifying Suitable Neighbors for Concurrent Transmissions in Point-to-Point Communications. In Proceddings of the ACM International Conference on Modeling, Analysis and Simulation of Wireless and Mobile Systems (MSWiM). 